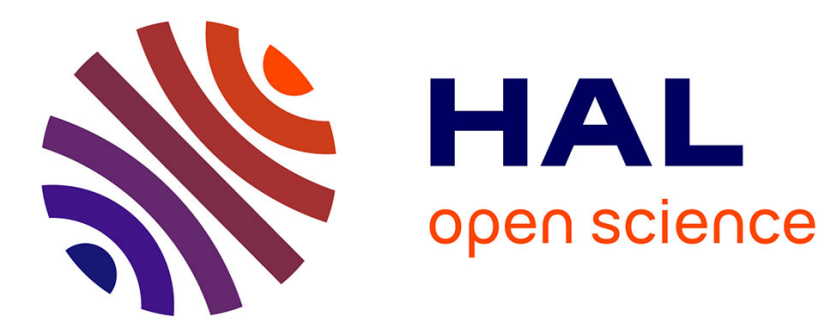

\title{
Optical power management using second harmonic generation in periodic segmented waveguides
}

Pierre Aschieri, Fabrizio Fogli, Pierre Aumont, M. de Micheli, Gaetano Bellanca, Paolo Bassi

\section{- To cite this version:}

Pierre Aschieri, Fabrizio Fogli, Pierre Aumont, M. de Micheli, Gaetano Bellanca, et al.. Optical power management using second harmonic generation in periodic segmented waveguides. Optics Communications, 2000, pp.55-61. 10.1016/j.optcom.2004.02.049 . hal-00460759

\section{HAL Id: hal-00460759 https://hal.science/hal-00460759}

Submitted on 2 Mar 2010

HAL is a multi-disciplinary open access archive for the deposit and dissemination of scientific research documents, whether they are published or not. The documents may come from teaching and research institutions in France or abroad, or from public or private research centers.
L'archive ouverte pluridisciplinaire HAL, est destinée au dépôt et à la diffusion de documents scientifiques de niveau recherche, publiés ou non, émanant des établissements d'enseignement et de recherche français ou étrangers, des laboratoires publics ou privés. 


\title{
Optical power management using second harmonic generation in periodic segmented waveguides
}

\author{
Pierre Aschiéri ${ }^{\mathrm{a}}$, Fabrizio Fogli ${ }^{\mathrm{b}}$, Pierre Aumont ${ }^{\mathrm{a}}$, \\ Marc De Micheli ${ }^{b}$, Gaetano Bellanca ${ }^{c}$, Paolo Bassi ${ }^{\mathrm{d}}$ \\ ${ }^{\mathrm{a}}$ Laboratoire de Physique de la Matière Condensée \\ Université de Nice-Sophia Antipolis \\ Parc Valrose, Nice, France \\ ${ }^{\mathrm{b}}$ Moduloptic S.A. Technopôle de Sophia Antipolis \\ 2720 Chemin de St. Bernard \\ 06220 Vallauris, France \\ ${ }^{\mathrm{c}}$ Dipartimento di Ingegneria \\ University of Ferrara \\ Via Saragat 1, I 44100 Ferrara, Italy \\ ${ }^{\mathrm{d}}$ Dipartimento di Elettronica Informatica e Sistemistica \\ University of Bologna \\ Viale Risorgimento 2, I 40136 Bologna, Italy
}

\begin{abstract}
A combination of loss properties of Periodic Segmented Waveguides and second order wavelength conversion processes is shown to exhibit features which allow to realize an adjustable optical power limiter using classical integrated optics technologies. Results of numerical simulations are presented to confirm the viability of the proposed approach using input and output power levels in the range involved in standard optical telecommunication systems.
\end{abstract}

Key words: Segmented waveguides, Optical power limiting, Second Harmonic Generation, Propagation losses, Phase matching, Integrated optics PACS: 42.82.-m, 42.65.K,

\section{Introduction}

The possibility of performing all optical signal processing in systems of the new generation requires devices with advanced functionalities. Optical limiting is 
certainly one of these. Its importance is due not only to the fact that optical logic elements have become more and more attractive, but also to the fact that the increased power of optical sources and the widespread use of optical amplifiers has made power limiting or power controlling necessary.

Many mechanisms have been used so far to obtain such devices as shown, for example, in [1-6] and references therein. Various materials, such as ferroelectric, semiconductor and organic ones, have been considered as well. The majority of the studied devices exploits third order non linear effects, which have, however, two main drawbacks. The former is related to the fact that this kind of non linear effects requires high optical intensities or materials with large Kerr coefficients, such as organic ones or PTS, DAN or Optimized Stilbene [7]. The latter comes as a consequence of the insertion of these devices in standard integrated optical circuits, which leads to hybrid and not monolithic solutions, with an impact in the overall device mechanical stability and the technological process.

In this paper we propose a different approach, able to solve both of these problems. It is still based on non linear effects, but of the second order type. As these effects can be easily generated in waveguides fabricated in substrates used in standard optical integrated circuits (mostly Lithium Niobate), this kind of optical limiter can be easily inserted in monolithic integrated optical devices. Moreover, limiting action is possible even with low power signals, overcoming one of the cited drawbacks of similar devices based on third order non linearities.

The use of second order non linear effects to obtain optical limiters had been previously proposed by Conti $e$ t al. [8]. In that case, the propagating beam was attenuated by coupling with a back propagating Second Harmonic (SH) signal generated in a suitable periodic structure. This, however, makes an in-line absorber necessary to eliminate such spectral component and avoid possible undesired effects in the whole optical system. In the device we propose, which will be illustrated in the next section together with its operating principles, light attenuation will be provided by the device itself, thus eliminating the need of that extra component. Preliminary numerical results will then be presented and discussed to show the viability of the approach. Finally, conclusions will be drawn.

\section{The optical power limiting device}

The optical limiter behavior we propose takes advantage of the combination of both linear and non linear effects on the propagating beam due to two different and independent periodicities. The device consists, in fact, in a Periodic 
Segmented Waveguides (PSW), a waveguide with longitudinally periodic characteristics [9], fabricated on a periodically poled Lithium Niobate substrate, as sketched in figure 1 . The substrate crystal, with linear refractive index $n_{s}$, is periodically poled with period $\Lambda_{Q P M}$. In this substrate, a PSW is diffused with period $\Lambda_{P S W}$ and refractive index of the guiding part equal to $n_{g}>n_{s}$.

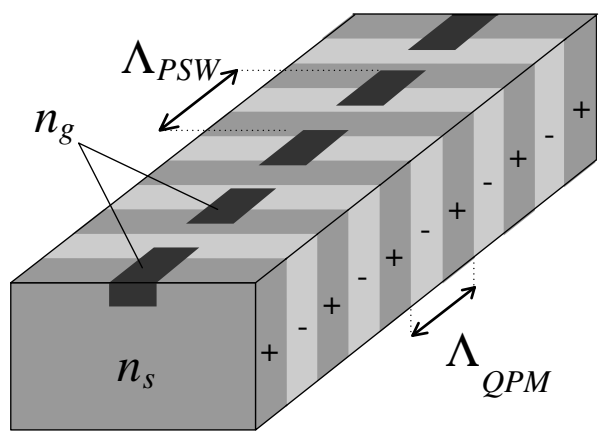

Fig. 1. PSW of a period $\Lambda_{P S W}$ embedded in a $\chi^{(2)}$ periodically poled substrate with period $\Lambda_{Q P M}$, being $n_{s}$ and $n_{g}$ the optical indices of the substrate and the guiding segments respectively such as $n_{s}<n_{g}$

To illustrate the working principle of this device, it is convenient to start considering the linear PSW alone. It has been shown in the past years that this kind of guides exhibits several interesting properties. For example, they have been used to fabricate tapers or mode filters [9-11], taking advantage of the fact that the mode spot size and propagation constant depend on the Duty-Cycle (DC), defined as the ratio of the high index segment over the period $\Lambda_{P S W}$. The PSW feature, which is of main interest for this paper, concerns the spectral behavior of the intrinsic losses, which depend on the waveguide opto-geometrical parameters. As shown by Weissman et al. [9], in fact, the positions and the number of maxima and minima of attenuation can be tailored to specific needs by a proper choice of the waveguide parameters such as its period, width or depth, resulting in spectral attenuation plots with behavior similar to that reported in figure 2. Considering that plot, however, one can notice a peculiar feature that can be exploited for the realization of a power limiter based on $\chi^{(2)}$ non linear effects. The values of the attenuation coefficient can be in fact very small (about $0.1 \mathrm{~cm}^{-1}$ ) for a wavelength (in this case $\lambda=1.6 \mu \mathrm{m})$ and sufficiently high $\left(4.2 \mathrm{~cm}^{-1}\right)$ for half that wavelength, in this case $\lambda=0.8 \mu \mathrm{m}$, i.e. at the double frequency. Moreover, different wavelengths and relevant attenuation values can be easily tailored, depending on specific purposes, by a proper design of the PSW geometrical characteristics $[12]$.

It comes then easy to imagine a power limiting effect related to the possibility of transferring part of the exceeding power from the almost lossless frequency to the double, lossy, one. This can be done taking advantage of second order non linear effects which can take place in periodically poled materials, i.e. materials in which the sign of the second order nonlinear coefficient $\chi^{(2)}$ is 


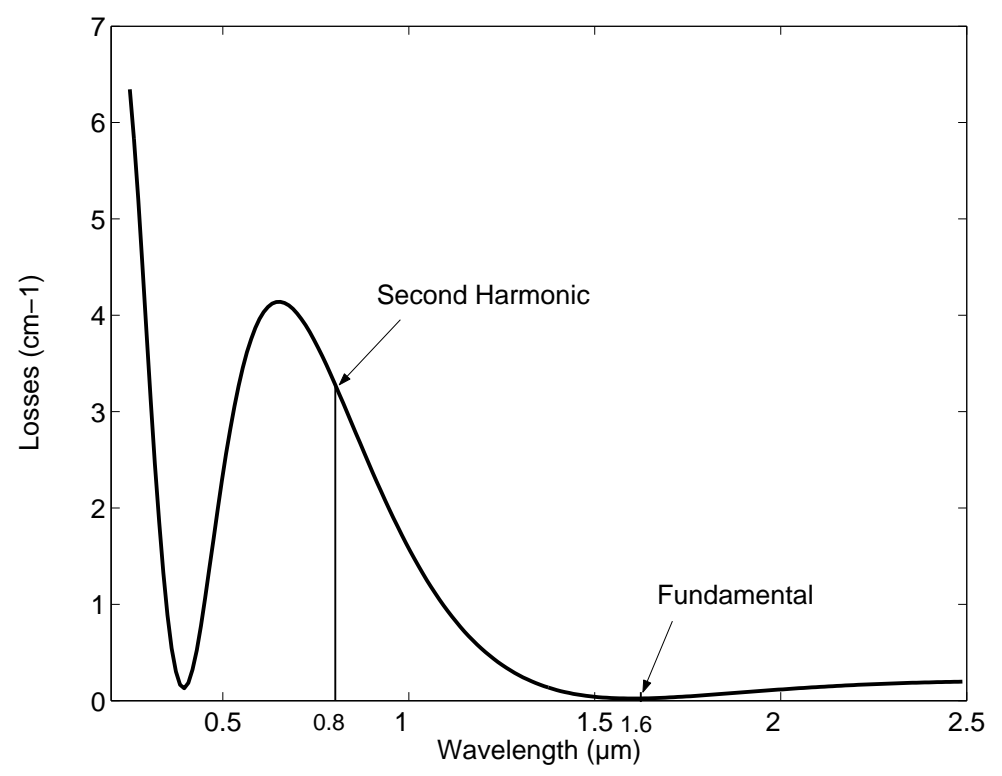

Fig. 2. Losses as a function of the wavelength in a PSW [12], segmentation period is $\Lambda_{P S W}=10 \mu \mathrm{m}$.

changed with the period we have previously called $\Lambda_{Q P M}$. Letting $k_{s h}=2 \pi / \lambda_{s h}$ the wave number of the Second Harmonic (SH) wave and $k_{f}=2 \pi / \lambda_{f}$ that of the fundamental one, with wavelengths respectively equal to $\lambda_{s h}$ and $\lambda_{f}$, if the condition

$$
2\left(k_{s h}-k_{f}\right)-\frac{2 \pi}{\Lambda_{Q P M}}=0
$$

is satisfied, the periodic structure guarantees the fulfillment of the matching condition between the fundamental wave and its $\mathrm{SH}$ one. This means that a complete power transfer between them can be obtained. If exact phase matching cannot be achieved, efficient nonlinear wavelength conversion has been anyway obtained, in materials such as $K T P$ or $\mathrm{LiNbO}_{3}$, using the Quasi Phase Matching (QPM) or Balanced Phase Matching (BPM) schemes [13,14]. These results have been obtained considering modes propagating both in bulk materials [15-17] and in waveguides [18] realized in those substrates.

In conclusion, the power limiting action of the device illustrated in figure 1 can be achieved designing the linear PSW almost lossless for a given wavelength and lossy at half that wavelength. The required wavelength conversion, which transfers some power from the lossless frequency to the lossy one, can be obtained using a Second Harmonic Generation (SHG) process. To achieve a phase matching condition between the fundamental and the Second Harmonic wave, the periodic poling of the substrate can be used to guarantee the Quasi Phase Matching (QPM) condition, widely used in the guiding configuration [18]. Note that the efficiency of the wavelength conversion and the amount 
of losses in the device vary as a function of the optical power injected in the waveguide. The device will then act as an adjustable optical limiter since the increase of losses, and the relevant reduction of the output power, is a function of the input power. The possibility of using Lithium Niobate to fabricate such a device provides the further advantage of allowing simple integration of the limiter with other optical components with different functionality on the same substrate.

\section{Results}

This section reports the results of some numerical simulations, which confirm the viability of the proposed approach. Such a preliminary analysis has been developed using a 2D structure, which is certainly simpler to study, but is nevertheless a good approximation of the real 3D case. Full 3D modelling will be however required for the final device design. For simplicity, a TE polarization has also be assumed for the fields at the fundamental and second harmonic frequencies. Using the classical assumption of the slowly envelope variation and neglecting backward propagating waves, the coupled parabolic wave equations representing the evolution of the two fields in a planar PSW are then the following ones [19]:

$$
\begin{aligned}
& 2 j k_{0} n_{0} \frac{\partial E_{f}}{\partial z}=\frac{\partial^{2} E_{f}}{\partial x^{2}}+k_{0}^{2}\left(n_{f}^{2}(x, z)-n s_{f}^{2}\right) E_{f}+k_{0}^{2} \chi^{(2)} e^{-j \Delta k z} E_{s h} E_{f}^{*} \\
& j k_{0} n_{0} \frac{\partial E_{s h}}{\partial z}=\frac{\partial^{2} E_{s h}}{\partial x^{2}}+k_{0}^{2}\left(n_{s h}^{2}(x, z)-n s_{s h}^{2}\right) E_{s h}+k_{0}^{2} \chi^{(2)} e^{-j \Delta k z} E_{f} E_{f}
\end{aligned}
$$

where $E_{s h}$ and $E_{f}$ are the fields of the $\mathrm{SH}$ and the fundamental wave respectively, $n s_{f}$ and $n s_{s h}$ are the $\mathrm{LiNbO}_{3}$ substrate indexes for the $\mathrm{SH}$ and the fundamental wave respectively, used here as reference indexes [19], $k_{0}=2 \pi / \lambda_{f}$ is the free space number at the fundamental frequency. Finally, $\Delta k=2\left(k_{s h}-k_{f}\right)$ is the phase mismatch between the fundamental and the SH. The refractive indexes of the PSW are $n_{s h}(x, z)$ and $n_{f}(x, z)$ for the SH and the fundamental wave respectively. They are periodic in $z$ in the waveguide core with period $\Lambda_{P S W}$. Moreover, the second order nonlinear susceptibility of the material $\chi^{(2)}$ is periodic with a period $\Lambda_{Q P M}$.

Equations (2) can be solved using a Non Linear Beam Propagation Method (NL-BPM) using a Leap-Frog scheme $[20,21]$ which allows to introduce second order nonlinear effects. These equations provide mode field amplitudes and transversal field shape evolution along the longitudinal direction. If one is concerned only with amplitude coefficients, but not in the computation of the transversal field shape, a simpler and faster one dimensional model can be 
considered. The basic coupling equations reduce then to the following ones:

$$
\begin{aligned}
& \frac{d E_{f}}{d z}=-\frac{j k_{0}^{2} \chi^{(2)}}{2 n_{f}} E_{s h} E_{f}^{*} e^{-j \Delta k z}-\alpha_{f} E_{f} \\
& \frac{d E_{s h}}{d z}=-\frac{j k_{0}^{2} \chi^{(2)}}{2 n_{s h}} E_{f} E_{f} e^{-j \Delta k z}-\alpha_{s h} E_{s h} .
\end{aligned}
$$

These equations can be solved using a Runge Kutta 4th order solver (RK4) [22] which is faster to run. In this unidimensional model, propagation losses have been introduced using the coefficients $\alpha_{f}$ and $\alpha_{s h}$ which take into account the losses associated with the fundamental and the $\mathrm{SH}$ signals respectively.

In the following, physical parameters of classical periodically poled $\mathrm{LiNbO}_{3}$ PSW's are used in the numerical simulations: $\lambda_{f}=1.6 \mu \mathrm{m}, \lambda_{s h}=0.8 \mu \mathrm{m}, n_{f}=$ 2.13595, $n_{s h}=2.17536, \Lambda_{P S W}=10 \mu \mathrm{m}, \Lambda_{Q P M}=18.8657 \mu \mathrm{m}$, the waveguide width is $d=2 \mu \mathrm{m}$ and the index difference between the substrate and the guiding segments $\Delta n=0.02$. The index profile distribution in the waveguide is assumed "step-like", as this is a good approximation of what results using the proton exchange technique [23] to fabricate waveguides in $\mathrm{LiNbO}_{3}$. With these parameters, a preliminary calculation of the propagation losses in the linear PSW shows that the fundamental and the $\mathrm{SH}$ wave attenuation coefficients result to be $\alpha_{f}=0.08 \mathrm{~cm}^{-1}$ and $\alpha_{s h}=3.72 \mathrm{~cm}^{-1}$ respectively.

If one includes second order non linear effects in the calculations, the power evolution along a $4 \mathrm{~mm}$ waveguide length computed with the NL-BPM and RK4 methods for the fundamental and the $\mathrm{SH}$ wave in the case of $31 \mathrm{~mW} / \mu \mathrm{m}$ input power turns out to be that reported in figure 3-a. For such a waveguide length, the input power does not cause an efficient $\mathrm{SH}$ wavelength generation and the input power for the fundamental does nor vary significantly, except a small attenuation due to intrinsic waveguide losses and some wavelength conversion to the $\mathrm{SH}$. Plots reported in the figure show also that there is a good agreement between the results obtained using the two different approaches.

If the input power is increased, the conversion efficiency to $\mathrm{SH}$ is expected to improve and, then, also losses at the fundamental wavelength should rise accordingly, thus reducing the corresponding output power. This happens, as it is shown in figure 3-b, which reports results calculated when the injected power is equal to $4 \mathrm{~W} / \mu \mathrm{m}$. Agreement between the results of the two methods is still good, though there is a slight discrepancy between the curves after $1000 \mu \mathrm{m}$ propagation length. However these differences, which are probably due to the intrinsic differences between the two approaches, do not seem dramatic in this context. We are, in fact, just trying to show the existence of an effect (which is confirmed by both approaches) and not designing a real device. Moreover, as previously mentioned, 2D modelling alone cannot be used for the device design, but only to find the starting set of parameters to be used in the final 
3D optimization.

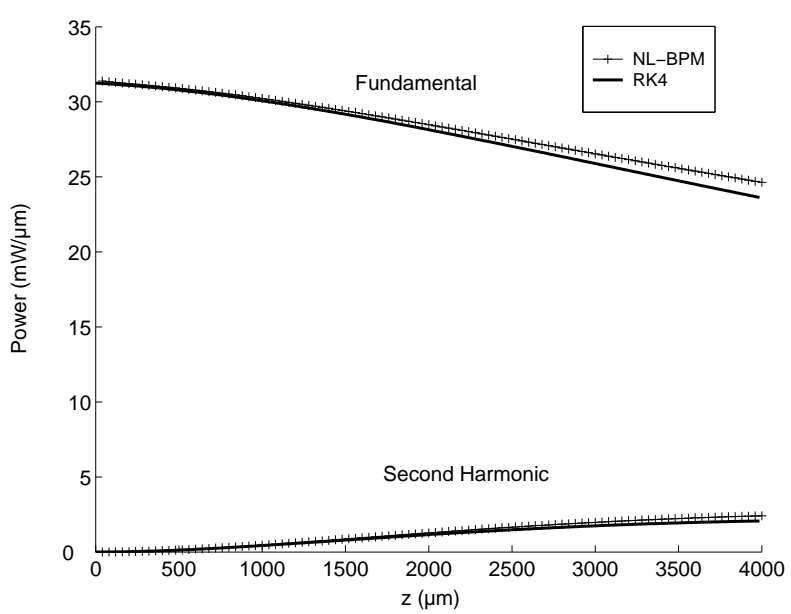

(a)

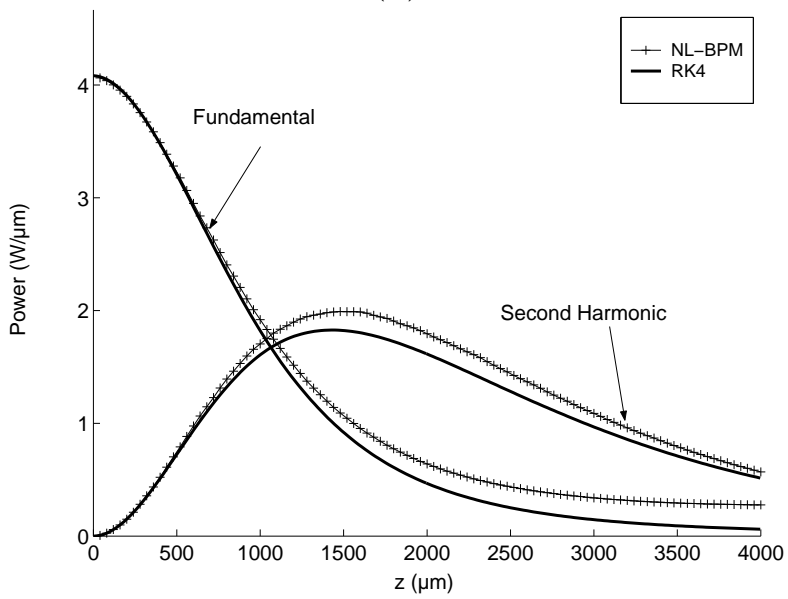

(b)

Fig. 3. Power evolution in the fundamental and $\mathrm{SH}$ wave for a low (a) and a high (b) input power injected in the waveguide [24].

Finally, figures 4-a and 4-b report the results corresponding to fundamental and SH mode transversal field intensity distributions in the waveguide in the case of an high input power. One can note that for a high input power, while the intensity associated with the fundamental wavelength decreases due to wavelength conversion process, the $\mathrm{SH}$ intensity increases with the propagation but it is attenuated at the same time because of the propagation losses for this wavelength. These plots also highlight the cause of this high attenuation, which is related to the strong light coupling into radiation modes of the waveguide.

An overall view of the device behavior as optical limiter can be obtained considering the device input-output characteristics in terms of field intensities at the fundamental and the second harmonic frequencies. For the device considered so far, by solving equations (3), the plots presented in figure 5 are obtained: the limiting effect is evident, as the transmission curve is not linear. 


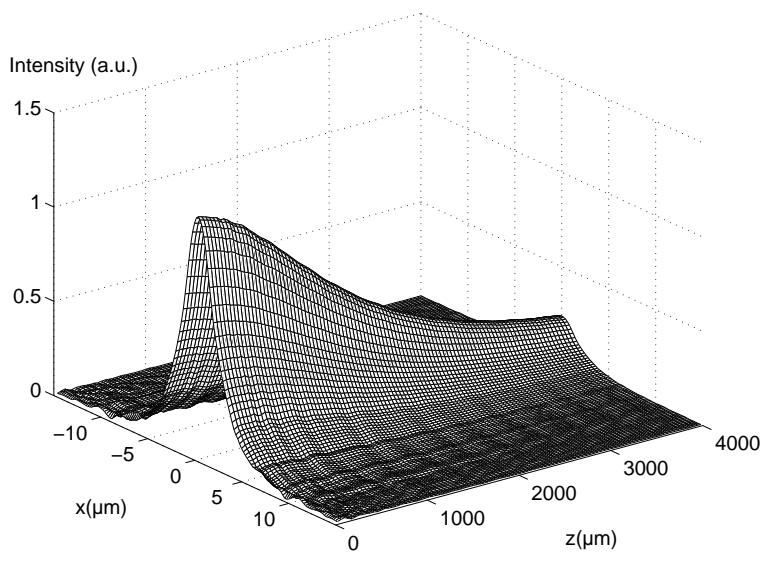

(a)

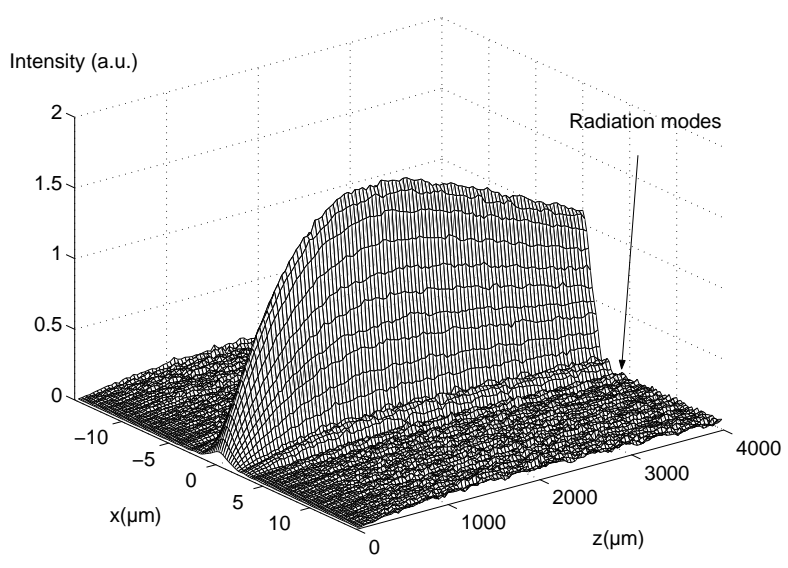

(b)

Fig. 4. Intensity mode profile evolution in the waveguide for the fundamental (a) and $\mathrm{SH}$ wave (b) for an high input power

The intensity plots shown so far have been determined using a set of geometrical parameters for the PSW and the periodic poling. Optimization of the device to obtain the desired features in terms of conversion efficiency and $\mathrm{SH}$ loss must be done by a proper choice of the waveguide parameters. For example, a reduction of the device length or a reduction of losses at the $\mathrm{SH}$ will increase the transmitted power. Furthermore a change of the PSW characteristics will change also the attenuation coefficient of the $\mathrm{SH}$ signal and so on. So, to fully include all the propagation effects, including radiation losses, 2D modelling can be considered only the preliminary stage of a full 3D device design. But, as mentioned before, here we want only to demonstrate the feasibility of the newly conceived device. Despite these limitations, however, 2D modelling allows also to show a further interesting feature of this device. It can also be observed, in fact, that some tuning of the device characteristics is possible even after its fabrication. This may be useful, for example, to reduce 


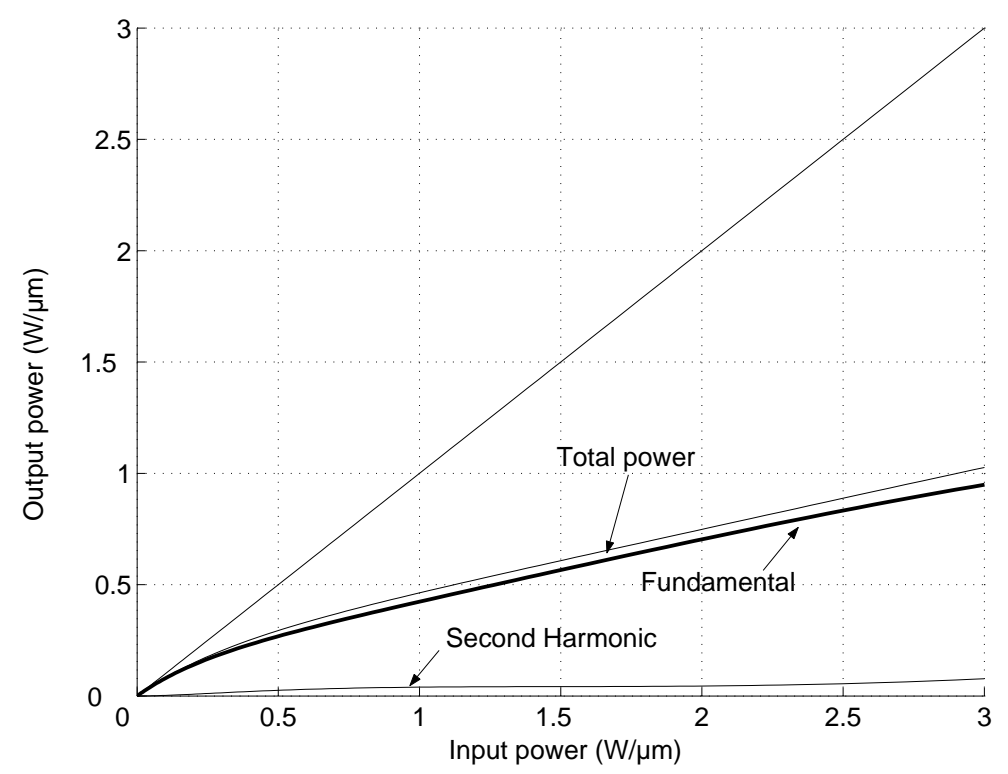

Fig. 5. Transmission efficiency of the device as a function of the input power

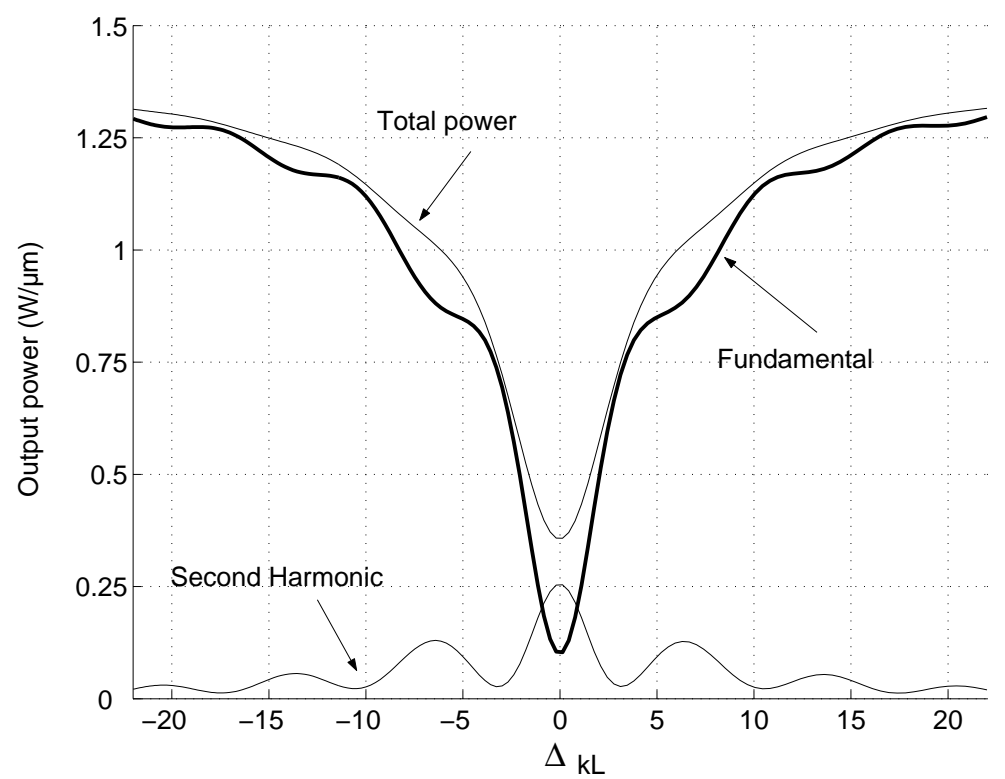

Fig. 6. Output power as a function of the phase mismatched $\Delta k L$.

the effect of fabrication tolerances or for fine tuning of the device. A further degree of freedom is given by the possibility of changing the output power level varying the phase mismatch $\Delta k$. Figure 6 reports results concerning the transmitted output power of the previous waveguide for a given input power of $1.5 \mathrm{~W} \mu \mathrm{m}$ as a function of the detuning $\Delta k L$. Results show that power management is possible if an adjustment of the phase mismatch is realized. The $\Delta k L$ required for an efficient power tuning is very narrow and easily adjustable with the temperature or with an electro-optical system in materials like $\mathrm{LiNbO}_{3}$. For example a $\Delta k L=2.5$ changes the output power from 0.25 
$\mathrm{W} / \mu \mathrm{m}$ to $0.75 \mathrm{~W} / \mu \mathrm{m}$, which corresponds to a temperature variation of $15 \mathrm{~K}^{\circ}$ at room temperature in $\mathrm{LiNbO}_{3}$.

\section{Conclusions}

In this paper, a new optical limiter structure is proposed. It is based on second order non linear effects in periodically segmented waveguides fabricated in a periodically poled Lithium Niobate substrate. The linear Periodically Segmented Waveguides is designed in such a way that it is almost transparent for the fundamental wavelength and dissipative for its Second Harmonic. Second order non linear effects, related to the periodic poling of the substrate, guarantee an efficient phase matching condition between the input signal wavelength and the Second Harmonic one. Optical limiting characteristics depend on the conversion efficiency between the fields propagating at the almost lossless fundamental wavelength and the lossy Second Harmonic, which varies according to the input signal power. Preliminary 1D and 2D numerical analysis has been performed and results confirm that the proposed device can be used as a versatile optical power management tool in the guided optics domain. From a technological point of view, no major difficulty seems to exist as the device is based on a well known and widely used technologies which, moreover, guarantee easy integration of this component in more complex integrated optical circuits.

\section{Acknowledgments}

Part of this work has been funded by the Italian Ministry of Education, University and Research (MIUR).

\section{References}

[1] A. Mecozzi, S. Trillo, S. Wabnitz, "Spatial instabilities, all-optical limiting, and thresholding in nonlinear distributed-feedback devices", Opt. Lett., vol. 1, no. 12, pp. 979-1077, 1987.

[2] P. Aschieri, P. Baldi, L. Chanvillard, M. P. De Micheli, D. B. Ostrowsky, G Bellanca, P. Bassi, "Optical power limiting using nonlinear periodic segmented waveguides", Nonlinear Optics, vol. 21, pp. 503-513, 1999. 
[3] D. I. Kovsh, S. Yang, D. J. Hagan, E. W. Van Stryland, "Nonlinear Optical Beam Propagation for Optical Limiting", Applied Optics-LP, vol. 38, no. 24, pp. 5168-5180, August 1999.

[4] D. Pelinovsky, J. Sears, L. Brzozowski, E. H. Sargent, "Stable all-optical limiting in nonlinear periodic structures. I. Analysis", Journal of the Optical Society of America B-Optical Physics, vol. 19, no. 1, pp. 43-53, 2002.

[5] D. Pelinovsky, E. H. Sargent, "Stable all-optical limiting in nonlinear periodic structures. II. Computations", Journal of the Optical Society of America BOptical Physics, vol. 19, no. 8, pp. 1873-1889, 2002.

[6] W. N. Ye, L. Brzozowski, E. H. Sargent, D. Pelinovsky, "Stable all-optical limiting in nonlinear periodic structures. III. Nonsolitonic pulse propagation", Journal of the Optical Society of America B-Optical Physics, vol. 20, no. 4, pp. 695-705, 2003.

[7] G. I. Stegeman, " $\chi^{2}$ cascading: nonlinear phase shifts", Quantum Semiclass. Opt., vol. 9, pp. 139-153, 1997.

[8] C. Conti, G. Assanto, S. Trillo, "Cavityless oscillation through backward quasiphase-matched second-harmonic generation", Opt. Lett., vol. 24, no. 16, pp. 1139-1141, 1999.

[9] Z. Weissman, A. Hardy, "Modes of periodically segmented waveguides", IEEE J. of Lightwave Technology, vol. 11, pp. 1831-1838, 1993.

[10] M. H. Chou, M. A. Arbore, M. M. Fejer, "Adiabatically tapered periodic segmentation of channel waveguides for mode-size transformation and fundamental mode excitation", Opt. Lett., vol. 17, pp. 794-796, 1996.

[11] D. Ortega, J. M. Aldariz, J. M. Arnold, J. S. Aitchison, "Analysis of "Quasi Modes" in Periodic Segmented Waveguides", IEEE J. of Lightwave Technology, vol. 17, pp. 369-375, 1999.

[12] A Java applet simulation of propagation losses as a function of the wavelength in PSW is avalable at http://www.unice.fr/optics/psw_wl.html

[13] J. D. Bierlein, D. B. Laubacher, J. B. Brown, C. J. van der Poel, "Balanced phase matching in $\mathrm{KTiOP}_{4}$ waveguides", Appl. Phys. Lett., vol. 56, pp. 1725$1727,1990$.

[14] Z. Weissman, A. Hardy, M. Katz, M. Oron, D. Eger, "Second-harmonic generation in Bragg-resonant quasi-phase-matched periodically segmented waveguides", Opt. Lett., vol. 20, no. 7, pp. 674-676, 1995.

[15] J. A. Armonstrong, N. Bloembergen, J. Ducuing, P. S. Pershan, "Interactions between light waves in nonlinear dielectric", Phys. Rev., vol. 127, pp. 1918-1939, 1962 .

[16] M. Okada, K. Takizawa and S. Ieiri, "Second harmonic generation by periodic laminar structure of nonlinear optical crystal", Optics Communications, vol. 18, no. 3, pp. 331-334, 1976. 
[17] L. E. Myers, R. C. Eckardt, M. M. Fejer, R. L. Byer, W. R. Bosenberg, J. W. Pierce, "Quasi-phasematched optical parameteric oscillators using bulk periodically-poled LiNbO3," J. Opt. Soc. Am. B, 12, pp. 2102-2116, 1995.

[18] M. M. Fejer, G. A. Magel, D. H. Jundt, R. L. Byer, "Quasi-phase-matched second harmonic generation: Tuning and tolerances", IEEE J. Quantum Electronics, vol. 28, no. 11, pp. 2631-2654, 1992.

[19] H. F. Chou, C. F. Lin, G. C. Wang, "An iterative finite difference beam propagation method for modeling second-order nonlinear effects in optical waveguides", IEEE J. of Lightwave Technology, vol. 16, no. 9, pp. 1686-1693, 1998.

[20] J. C. Strikwerda, "Finite Differnce Scheme and Partial Differential Equations", Wadsworth, New York, 1989.

[21] P. S. Weitzman and U. Ostenberg, "A modified beam propagation method to model second harmonic generation in optical fiber", IEEE J. of Quantum Electronics, vol. 29, pp. 1437-1443, 1993.

[22] "Numerical recipies in C", Second Edition, Cambridge University Press.

[23] M. De Micheli, J. Botineau, S. Neveu, P. Sibillot, D. B. Ostrowsky, "Independent control of index and profile in proton-exchanged lithium niobate guide", Opt. Lett., vol. 8, pp. 114-115, 1983.

[24] A Java applet simulation of SH generation in PSW is avalable at http://www.unice.fr/optics/psw_shg.html 